\title{
Could readily silanized silica particles substitute silica coating and silanization in conditioning zirconium dioxide for resin adhesion?
}

Özcan, Mutlu ; Yetkiner, Enver

DOI: https://doi.org/10.1080/01694243.2015.1095628

Posted at the Zurich Open Repository and Archive, University of Zurich ZORA URL: https://doi.org/10.5167/uzh-128120

Journal Article

Accepted Version

Originally published at:

Ózcan, Mutlu; Yetkiner, Enver (2016). Could readily silanized silica particles substitute silica coating and silanization in conditioning zirconium dioxide for resin adhesion? Journal of Adhesion Science and Technology, 30(2):186193.

DOI: https://doi.org/10.1080/01694243.2015.1095628 
Could readily silanized silica particles substitute silica-coating and silanization in conditioning zirconium dioxide for resin adhesion?

\section{Mutlu Özcan, DDS, Dr.med.dent., PhD $^{d} /$ Enver Yetkiner, DDS, PhD ${ }^{b}$}

${ }^{a}$ Professor, University of Zurich, Dental Materials Unit, Center for Dental and Oral Medicine,

Clinic for Fixed and Removable Prosthodontics and Dental Materials Science, Zurich, Switzerland

${ }^{b}$ Associate Professor, Department of Orthodontics, Faculty of Dentistry, Ege University, Izmir, Turkey

Short title: Adhesion of resin cement to zirconia after silica particle deposition.

${ }^{*}$ Part of this study has been presented at the 91st General Session and Exhibition of the International Association for Dental Research (IADR), March, 20th-23rd, 2013, Seattle, Washington, USA.

Correspondance to: Prof. Dr. med. dent. Mutlu Özcan, University of Zürich, Dental Materials Unit, Center for Dental and Oral Medicine Clinic for Fixed and Removable Prosthodontics and Dental Materials Science, Plattenstrasse 11, CH-8032, Zürich, Switzerland. Tel: +41-44-63 45600, Fax: +41-44-63 44305. e-mail: mutluozcan@hotmail.com 
Abstract: Particle deposition with silica-coated alumina followed by silane application increase the physico-chemical interaction between the resin cement and zirconia. Unfortunately, due to hydrolytic degradation of the silane, achieved adhesion decreases after aging conditions. Recently, readily silanized silica particles became available, that eliminates silanization procedures. This study investigated the effect of particle types with different morphology and surface properties on the surface wettability and adhesion of resin cement to zirconia after aging. Zirconia specimens $\left(5 \times 5 \times 1 \mathrm{~mm}^{3}\right)$ (Emax ZirCAD, Ivoclar) were wet polished to 1200 grit silicone carbide abrasive and ultrasonically cleaned. Specimens were randomly assigned to one of the following surface conditioning protocols ( $N=36, n=9$ per group): Group CON: Control, no surface conditioning; Group AL: Chairside air-abrasion with aluminium trioxide $\left(50 \mu \mathrm{m} \mathrm{Al}_{2} \mathrm{O}_{3}\right)+$ air-drying + silane (Monobond S); Group SIL: Chairside air-abrasion with alumina particles coated with silica (SIL) (30 $\mu \mathrm{m}$ $\mathrm{SiO}_{2}$, SilJet) + air-drying + silane; Group 4: Chairside air-abrasion with readily silanized silica particles (SILP) (30 $\mu \mathrm{m} \mathrm{SiO}_{2}$, SilJet Plus). Adhesive resin (Heliobond) was applied and resin cement (Variolink II, Ivoclar) was bonded using polyethylene moulds and photopolymerized. The specimens were then submitted to thermocycling $\left(6.000\right.$ cycles, $5-55^{\circ} \mathrm{C}$, dwell time: $30 \mathrm{~s}$, transfer time: $5 \mathrm{~s}$ ). Shear bond test was performed using Universal Testing Machine $(1 \mathrm{~mm} / \mathrm{min})$. Pretest failures during thermocycling were considered $0 \mathrm{MPa}$. Failure modes were classified using optical microscope. In a separate set of specimens, contact angle measurements were performed ( $n=2 /$ group, sessile drop with water). Bond strength data (MPa) were analyzed using ANOVA and Tukey's post hoc tests $(\alpha=0.05)$. Twoparameter Weibull distribution values including the Weibull modulus, scale $(m)$ and shape (o), values were calculated. Contact angle measurements were in descending order as follows: SIL $\left(74^{\circ}\right)^{c}<\operatorname{CON}\left(60^{\circ}\right)^{c}<\operatorname{AL}\left(51^{\circ}\right)^{b}<\operatorname{SILP}\left(40^{\circ}\right)^{a}$. Bond strength (MPa) with SIL $(17.2 \pm 4)^{a}$ and SILP $(17.3 \pm 1.9)^{a}$ demonstrated no significant difference $(p>0.05)$, both being significantly higher than those of $A L(8.4 \pm 1.5)^{b}$ and $\operatorname{CON}(0)^{c}(p<0.05)$ after thermocycling. Failure types were exclusively adhesive in all groups. Weibull distribution presented lower 
shape (0) for AL (7.7) and SIL (5.3) and the highest for SILP (10.8). Silane coated silica particles (SilJet Plus) presented better wettability on zirconia than with alumina or alumina particles coated with silica and provided similar bond strength on zirconia obtained with silica coated and silanized surfaces. Since no silane application is needed with readily silanized silica particles, it may be used as a substitute for conventional silica coating and silanization, reducing the clinical procedures.

Keywords: Adhesion, air-abrasion, bond strength, resin cement, repair, silane coupling agent, surface conditioning, zirconia 


\section{Introduction}

Zirconium dioxide (hereon: zirconia) is a polycrystalline ceramic, free of silica that presents inferior adhesion of resin-based materials to this ceramic compared to its glass ceramic or metallic counterparts used as framework materials for fixed dental prosthesis (FDP). Adhesion of resin cements to zirconia becomes particularly essential for resin-bonded inlay or surface-retained FDPs since the retention of such reconstructions do not rely on mechanical retention. Thus, several surface conditioning methods have been suggested to condition zirconia surfaces physically and/or chemically where physico-chemical conditioning methods remain to be the best methods to increase adhesion of resin-based materials to zirconia [2-10].

Adhesion of resin cements to zirconia or repair resins could be improved by deposition of abrasives such as aluminium trioxide $\left(\mathrm{Al}_{2} \mathrm{O}_{3}\right)$ or alumina particles coated with silica (Rocatec Plus, CoJet, SilJet System) to zirconia followed by silanization [10]. In fact, abrasive particles vary in morphology and their impact on the zirconia surface works as a function of their morphology and other deposition parameters [11,13]. Small size silicacoated alumina particles with more favourable morphology was reported to result in less transformation of tetragonal phase into monoclinic $[12,13]$. Yet, bond strength results show dramatic decrease depending on the test type after hydrothermal aging which typically take place in thermocycling device at alternating temperatures between $5-55^{\circ} \mathrm{C}$ in water [10]. Aging of the adhesive joints is also partly due to hydrolytic instability of the silane coupling agents. Recently, silica particles have been introduced that are readily silanized, eliminating the silanization process, thereby also reducing the chairside procedures. It is not known to date whether such particles could substitute available abrasives used in conjunction with 
chairside air-abrasion procedures and exclude the additional application of silane coupling agents.

The objectives of this study therefore were to compare the effect of deposition of different particles on the a) surface wettability and b) adhesion of resin cement to zirconia after aging. The hypotheses tested were that particle type would not affect the surface wettability and adhesive strength of resin cement to zirconia.

\section{Materials and Methods}

Specimens, bonding procedures

Zirconia specimens $(5 \mathrm{~mm} \times 5 \mathrm{~mm} \times 1 \mathrm{~mm})$ (Emax ZirCAD, Ivoclar Vivadent, Schaan, Liechtenstein) were wet polished to 1200 grit silicone carbide abrasive (Struers $\mathrm{GmbH}$, Willich, Germany) and ultrasonically cleaned (Vitasonic II, Vita, Bad Säckingen, Germany) in distilled water for 5 minutes and allowed to dry at room temperature for 5 minutes. Specimens were randomly assigned to one of the following surface conditioning protocols $(\mathrm{N}=36, \mathrm{n}=9$ per group):

Group CON: No surface conditioning was performed in this group.

Group AL: The specimens were air-borne particle abraded using an intraoral air-abrasion device (Dento-Prep, RØNVIG, Daugaard, Denmark) with $50 \mu \mathrm{m} \mathrm{Al}_{2} \mathrm{O}_{3}$ (Korox, Bego, Bremen, Germany) perpendicular to the surface from a distance of approximately $10 \mathrm{~mm}$ for $20 \mathrm{~s} / \mathrm{cm}^{2}$ in circling motions at 2.8 bar for $20 \mathrm{~s}$ [14].

Group SIL: In this group, instead of ordinary alumina particles, $30 \mu \mathrm{m}$ alumina particles coated with silica $\left(\mathrm{SiO}_{2}\right)$ was used (SilJet, Danville Engineering, San Ramon, CA). Particle deposition protocol was practiced as described in Group AL.

Group SILP: In this group, pre-silanized $30 \mu \mathrm{m}$ alumina particles coated with silica $\left(\mathrm{SiO}_{2}\right)$ was used (SilJet Plus, Danville Engineering). Particle deposition protocol was practiced as described in Group AL. 


\section{Contact Angle Measurement}

Additional specimens ( $\mathrm{n}=2$ per group) were made and 5 contact angle measurements per specimen were performed using a camera-based goniometer (Easydrop Drop Shape Analysis System, Kruess, Hamburg, Germany). For this purpose, $0.1 \mu \mathrm{l}$ sessile drop of distilled water was placed on the specimen surface located on a movable table using a micro syringe (diameter: $1.1 \mathrm{~mm}, \mathrm{NE} 42, \mathrm{Kruess})$ at $25^{\circ} \mathrm{C}$ room temperature. The drop was illuminated from one side, and the camera from the opposite side captured the image of the drop. The image was then transferred to the computer and contact angle was determined with the software referring to distilled water as the substance liquid according to YoungLaplace method [15]:

$\Delta p=\delta .\left(1 / r_{1}+1 / r_{2}\right)$

where

$\Delta p$ is the difference in pressure between the outside of the drop and its inside.

$r_{1}$ and $r_{2}$ stand for the principle radii of the curvature.

Adhesion Tests and Failure Analysis

Following contact angle measurements, specimens in each group received one coat of silane coupling agent (Monobond S, Ivoclar Vivadent) and waited for 5 minutes for its reaction. Subsequently, one coat of adhesive resin (Heliobond, Ivoclar Vivadent) was applied with a microbrush, air-thinned and photo-polymerized for $40 \mathrm{~s}$ using a photopolymerization device (Demetron LC, $800 \mathrm{~mW} / \mathrm{cm}^{2}$, Kerr Corporation, Orange, CA). Resin cement (Variolink II, Ivoclar Vivadent) was then bonded using polyethylene moulds incrementally (height: $3 \mathrm{~mm}$; diameter: $3.5 \mathrm{~mm}$ ) and photo-polymerized from the top for $40 \mathrm{~s}$ and from 4 directions additionally for $40 \mathrm{~s}$ each. The specimens were then submitted to thermocycling $\left(6.000\right.$ cycles, $5-55^{\circ} \mathrm{C}$, dwell time: $30 \mathrm{~s}$, transfer time: $\left.5 \mathrm{~s}\right)$. Pre-test failures during thermocycling were considered $0 \mathrm{MPa}$.

For the bond test, all specimens were mounted in the jig of the Universal Testing Machine 
(Zwick ROELL Z2.5 MA 18-1-3/7, Ulm, Germany) and the shear force (crosshead speed: 1 $\mathrm{mm} / \mathrm{min}$ ) was applied to the bonded interface until failure occurred.

Failure modes were identified using optical microscope (Stemi 2000-C, Carl Zeiss, Göttingen, Germany) at x10 magnification. Scanning Electron Microscope (SEM) (JSM5500, Jeol, Tokyo, Japan) images were obtained from particles tested.

Statistical Analyses

A sample size of 9 in each group was calculated to have more than $80 \%$ power to detect a difference of 7.5 MPa between mean values. According to the two-group Satterthwaite t-test (Statistix 8.0 for Windows, Analytical Software Inc, Tallahassee, FL, USA) with a 0.05 twosided significance level, this assumes that for conditoning wirh $\mathrm{Al}_{2} \underline{\mathrm{O}}_{3}$ standard deviation is

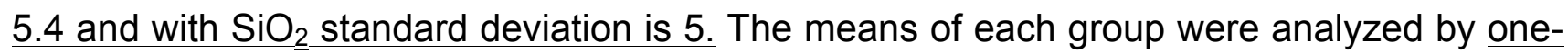
way analysis of variance (ANOVA) with shear bond strength (MPa) as the dependent variable and air-abrasion methods (3 levels: alumina, alumina particles coated with silica and pre-silanized silica) as the independent factors (Statistix 8.0 for Windows, Analytical Software Inc, Tallahassee, FL, USA). Multiple comparisons were made by Tukey`s post hoc tests. Also, for contact angle data, one-way ANOVA was used. $P$ values less than 0.05 were considered to be statistically significant in all tests.

\section{Results}

Significant difference was observed between the groups for both contact angle $(p<0.05)$ and bond strength $(p<0.05)$.

Contact angle measurements were significantly lower for SILP $\left(40^{\circ}\right)^{a}$ compared to those of other groups SIL $\left(74^{\circ}\right)^{c}$, $\operatorname{CON}\left(60^{\circ}\right)^{b}, \operatorname{AL}\left(51^{\circ}\right)^{b}$ (Fig. 1).

Bond strength (MPa) with SIL $(17.2 \pm 4)^{\mathrm{a}}$ and SILP $(17.3 \pm 1.9)^{\mathrm{a}}$ demonstrated no significant difference ( $p>0.05)$, both being significantly higher than those of $A L(8.4 \pm 1.5)^{b}$ and $\operatorname{CON}(0)^{c}$ $(\mathrm{p}<0.05)$ after thermocycling (Fig. 2). 
Weibull distribution presented lower shape (0) for AL (7.7) and SIL (5.3) and the highest for SILP (10.8) (Fig. 3).

Failure types were exclusively adhesive in all groups.

SEM images (x250) indicated rougher surface of individual alumina particles compared to silica coated alumina and pre-silanized silica (Figs. 4a-c).

\section{Discussion}

Since durable adhesion between zirconia and resin cements is still a clinical challenge, the present study evaluated adhesion to this ceramic after different chairside air-particle abrasion methods. Based on the results of this study with the pre-silanized silica particles, better surface wettability was obtained compared to air-abrasion with other particle types. Similarly, adhesion results were significantly better than with alumina air-abrasion and the control group but not significant with that of silica after thermocyling. Thus, null hypothesis could be rejected.

In general, surface wettability of zirconia the increases after air-particle abrasion, characterized by reduction in the contact angle measurements [16]. This property may be of importance for microleakage between the resin materials and zirconia.

Air-particle abrasion with alumina or silica particles increases the surface roughness, modifying the surface energy and wettability of zirconia, improving the micromechanical retention. Even though air-abrasion procedures may increase adhesion, hydrolytic degradation after aging may surpass the advantage gained from micromechanical retention [10]. The loss of all bonded specimens of the control group in thermocycle signifies the importance of air-abrasion surface conditioning. The particle morphology of alumina particles presents a more amorphous pattern than silica coated alumina ones or silica particles only where the latter does not yield to subsurface damage $[12,13]$. Silica coating leaves a fresh, chemically reactive silica (silicon dioxide) layer embedded on the 
conditioned surface [17], facilitating chemical covalent bonding with a silane coupling agent application.

Chemically activated silane coupling agents are hybrid inorganic-organic bifunctional molecules that are able to create a siloxane network with the hydroxyl $(\mathrm{OH})$ groups on the air-abraded surfaces and copolymerize with the hydrophobic resin matrix of the composite [17]. They have two ends: usually a methacrylate group (with vinylic carbon-carbon double bonds, $\mathrm{C}=\mathrm{C}$ ) and three hydrolysable ethoxy groups that during chemical activation turn into silanol groups $(\mathrm{Si}-\mathrm{OH})$. Silane monomers react with the silica-coated surface within the silanol groups forming hydrogen bonds and finally a covalently bonded very thin silane film. Silane film with its free carbon-carbon double bonds then reacts with the double bonds of resin composite luting cement [17]. Increased particle size and pressure may impair fatigue resistance or fit of the framework [12]. Thus, particles with small size and round morphology as in the case of silica particles are favorable. Similarly, silica is generally suggested to alumina because $-\mathrm{Si}-\mathrm{O}-\mathrm{Si}-\mathrm{O}-$ bonds are hydrolytically more stable than $-\mathrm{Al}-\mathrm{O}-\mathrm{Si}-[2]$. The results of this study also demonstrated almost two times less bond strength with alumina air-abrasion compared to groups conditioned with silica, supporting the hydrolysis phenomenon with alumina particles. Even though the bond strength with silica and silanization was similar to that of the pre-silanized silica coated group, the Weibull distribution indicated better reliability for the latter. This could be attributed to the hydrolysis of the silane coupling agent and thereby higher scatter in the data compared to presilanized silica coated groups where silane layer was coated on the particles using the sol gel technologies. Such a conditioning method would eliminate one clinical step but also decrease possibility of the problems associated with the short shelf life and hydrolysis of the silane coupling agent [18].

It has to be noted that in this study, the surfaces of the specimens were initially polished with 1200 grit silicone carbide abrasive. Typically, after milling process the surface of zirconia blocks have surface roughness of approximately $R_{\underline{a}}: 1.37 \mu \mathrm{m}$ and $R_{\underline{z}}: 8.8 \mu \mathrm{m}$ 
depending on the roughness of the milling burs and milling parameters [19]. Polishing with silicone carbide abrasive or air-abrasion certainly eliminates the initial roughness and grooves that occur during milling process, resulting in a smoother surface. This could explain the sole incidence of adhesive failures. On the other hand, the result in the control group corresponds well with the results of many previous studies as noted in a recent systematic review [10]. The bond results of this study using a methacrylate based cement after physicochemical activation of the surface in $A L(8.4 \mathrm{MPa})$ group after thermocycling is also in accordance with the results of this recent systematic review where $10.1 \mathrm{MPa}$ was reported with either alumina or silica coating [10]. In that respect, the results obtained in SIL (17.2 MPa) and SILP (17.3 MPa) groups, could be considered higher than this value but in the same range with phosphate monomer containing cements (16.85 MPa) [10].

Future studies should also consider long-term water storage as an aging medium and utilizing other cements to test the durability of the bond to zirconia after air-abrasion with pre-silanized particles. Yet, the absence of cohesive failures in the zirconia substrate and the high incidence of adhesive failures, truly denote poor quality of adhesion to zirconia even after air-abrasion with all types particles tested.

\section{Conclusions}

From this study, the following could be concluded:

1. Pre-silanized silica particles presented better wettability on zirconia compared to alumina and alumina particles coated with silica.

2. Readily silanized silica particles presented bond strength of resin cement to zirconia similar to silica coated and silanized surfaces but failure types were adhesive in all groups.

3. Weibull distribution indicated better reliability with pre-silanized silica particles compared to other particle types tested.

\section{Clinical Relevance}


Considering wettability, bond results and Weilbull characteristics, since no silane application is needed, readily silane coated silica particles may be advised as a substitute for conventional silica coating or air-abrasion with alumina, providing that all failures were adhesive between the cement and zirconia.

\section{Conflict of interest}

The authors did not have any commercial interest in any of the materials used in this study.

\section{Acknowledgements}

The authors would like to acknowledge Albert Trottmann, CDT for preparing the zirconia specimens, and Danville Engineering, San Ramon, USA for generous provision of the SilJet and SilJet Plus particles. 


\section{References}

1. Molin MK, Karlsson SL. Five-year clinical prospective evaluation of zirconia-based Denzir 3-unit FPDs. Int J Prosthodont 2008;21:223-227.

2. Özcan M, Vallittu PK. Effect of surface conditioning methods on the bond strength of luting cement to ceramics. Dent Mater 2003;19:725-731.

3. Matinlinna JP, Heikkinen T, Özcan M, Lassila LV, Vallittu PK. Evaluation of the resin adhesion to zirconia ceramic using some organosilanes. Dent Mater 2006; 22:824-31.

4. Özcan M, Kerkdijk S, Valandro LF. Comparison of resin cement adhesion to Y-TZP ceramic following manufacturers' instructions of the cements only. Clin Oral Investig 2008;12:279-282.

5. May LG, Passos SP, Capelli DB, Özcan M, Bottino MA, Valandro LF. Effect of silica coating combined to a MDP-based primer on the resin bond to Y-TZP ceramic. J Biomed Mater Res B Appl Biomater 2010;95:69-74.

6. Azimian F, Klosa K, Kern M. Evaluation of a new universal primer for ceramics and alloys. J Adhes Dent 2012;14:275-282.

7. Cura C, Özcan M, Isik G, Saracoglu A. Comparison of alternative adhesive cementation concepts for zirconia ceramic: glaze layer vs zirconia primer. J Adhes Dent 2012;14:75-82.

8. Everson P, Addison O, Palin WM, Burke FJT. Improved bonding of zirconia substructures to resin using a "glaze-on" technique. J Dent 2012;40:347-351.

9. Amaral M, Belli R, Cesar PF, Valandro LF, Petschelt A, Lohbauer U. The potential of novel primers and universal adhesives to bond to zirconia. J Dent 2014;42:90-98.

10. Özcan M, Bernasconi M. Adhesion to zirconia used for dental restorations: a systematic review and meta-analysis. J Adhes Dent 2015;17:7-26. 
11. Sato H, Yamada K, Pezzotti G, Nawa M, Ban S. Mechanical properties of dental zirconia ceramics changed with sandblasting and heat treatment. Dent Mater $\mathrm{J}$ 2008;27:408-414.

12. Özcan M, Melo RM, Souza RO, Machado JP, Valandro LF, Bottino M. Effect of airparticle abrasion protocols on the biaxial flexural strength, surface characteristics and phase transformation of zirconia after cyclic loading. J Mech Behav Biomed Mater 2013;20:19-28.

13. Souza ROA, Valandro LF, Melo RM, Machado JPB, Bottino MA, Özcan M. Airparticle abrasion on zirconia ceramic using different protocols: Effects on biaxial flexural strength after cyclic loading, phase transformation and surface topography. J Mech Behav Biomed Mater 2013;26:155-163.

14. Özcan M, Raadschelders J, Vallittu P, Lassila L. Effect of particle deposition parameters on silica coating of zirconia using a chairside air-abrasion device. J Adhes Dent 2013;15:211-214.

15. Strom G, Frederiksson M, Stenius P, Radoev B. Kinetics of steady-state wetting. J Colloid Interface Sci 1990;134:107-115.

16. Pereira Lde L, Campos F, Dal Piva AM, Gondim LD, Souza RO, Özcan M. Can application of universal primers alone be a substitute for airborne-particle abrasion to improve adhesion of resin cement to zirconia? J Adhes Dent 2015;17:169-174.

17. Matinlinna JP, Lassila LV, Özcan M, Yli-Urpo A, Vallittu PK. An introduction to silanes and their clinical applications in Dentistry. Int J Prosthodont 2004;17:155-164.

18. Özcan M, Matinlinna JP, Vallittu PK, Huysmans MC. Effect of drying time of 3methacryloxypropyltrimethoxysilane on the shear bond strength of a composite resin to silica-coated base/noble alloys. Dent Mater 2004;20:586-590.

19. Fraga S, Valandro LF, Bottino MA, May LG. Hard machining, glaze firing and hydrofluoric acid etching: Do these procedures affect the flexural strength of a leucite glassceramic? Dent Mater 2015;31:e131-140. 


\section{Captions to legends:}

\section{Figures:}

Fig. 1 Contact angle measurements after air-borne particle abrasion of zirconia (CON: Control, no air-abrasion; $\mathrm{AL}$ : $50 \mu \mathrm{m} \mathrm{Al}_{2} \mathrm{O}_{3}$, SIL: $30 \mu \mathrm{m}$ alumina particles coated with silica, SILP: Pre-silanized $30 \mu \mathrm{m}$ alumina particles coated with silica).

Fig. 2 Bond strength (MPa) of methacrylate based resin cement to zirconia after air-borne particle abrasion with different particle types. See Fig. 1 for group descriptions.

Fig. 3 Probability plot with Weibull curves $(95 \% \mathrm{Cl})$ using maximum likelihood estimation, scale and shape values for all groups.

Figs. 4a-c Scanning Electron photomicrographs of a) $\mathrm{Al}_{2} \mathrm{O}_{3}$ (x250), b) Siljet Sand (x250) and c) Siljet Plus (x250) particles. Note the rough and sharp morphology of surface of individual $\mathrm{Al}_{2} \mathrm{O}_{3}$ particles compared to silica coated $\mathrm{Al}_{2} \mathrm{O}_{3}$ particles in $\mathrm{b}$ and $\mathrm{c}$. 
Contact Angle Measurements

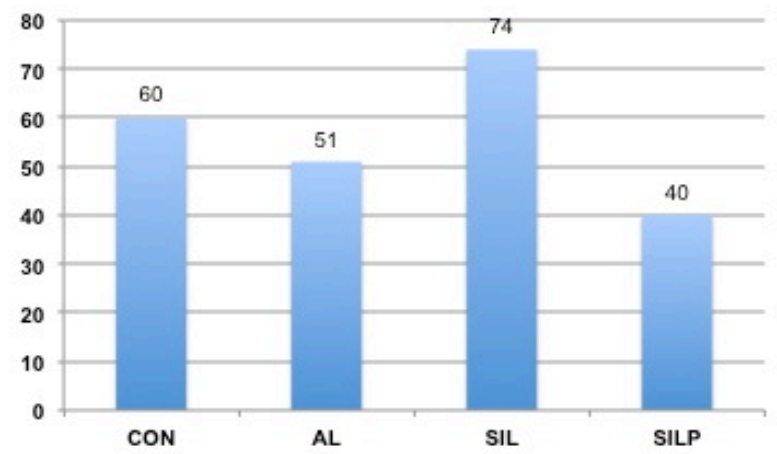

Figure 1

Shear Bond Strength (MPa)

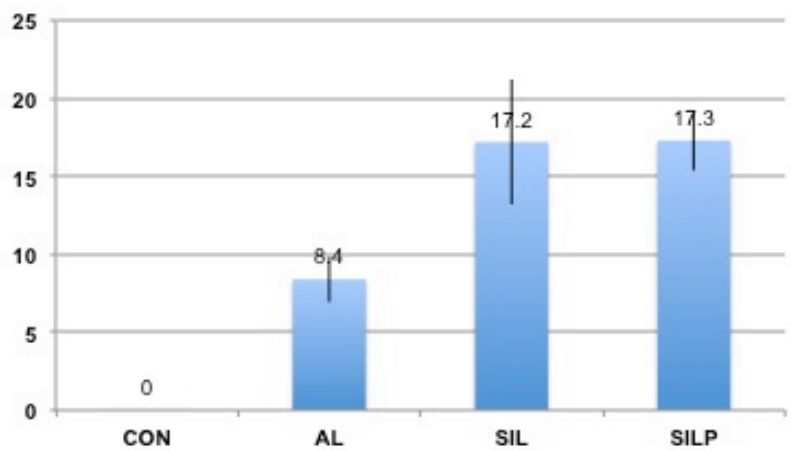

Figure 2

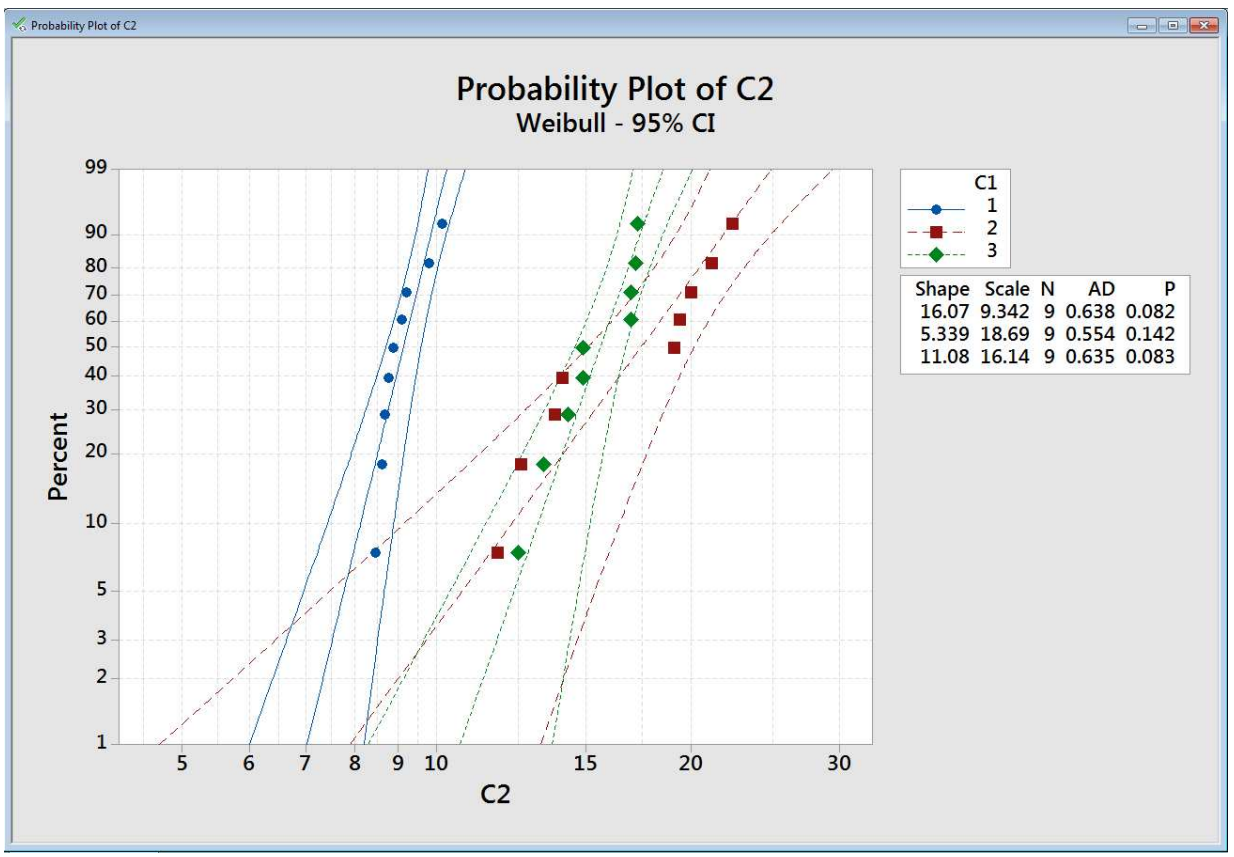

Figure 3 


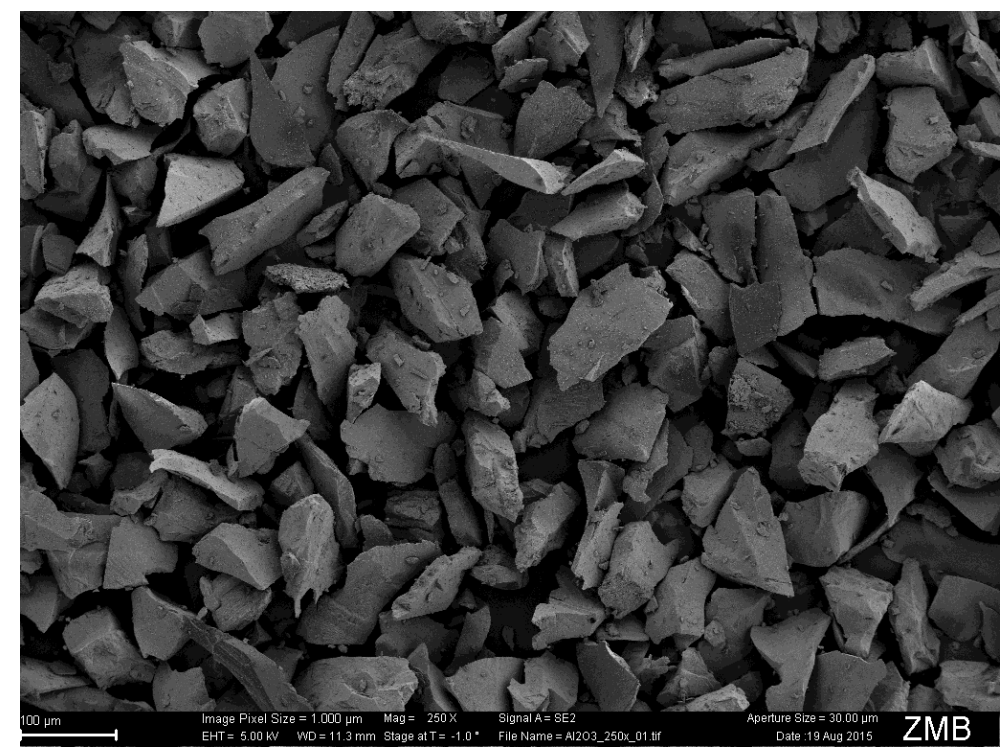

Figure 4a

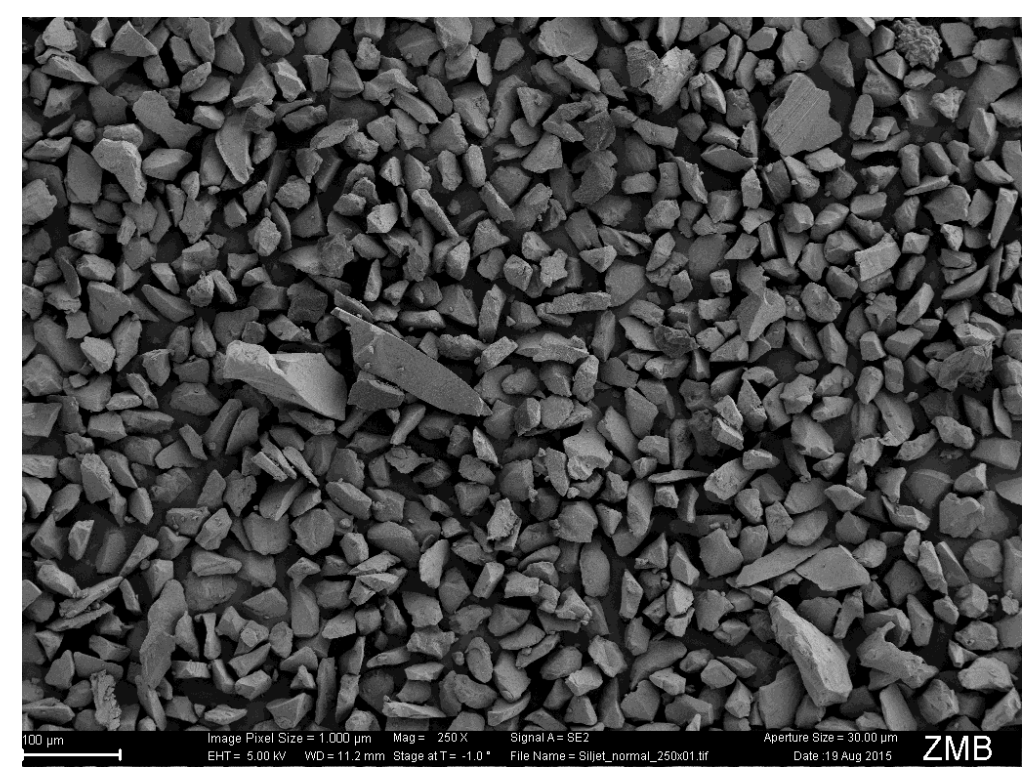

Figure $4 b$

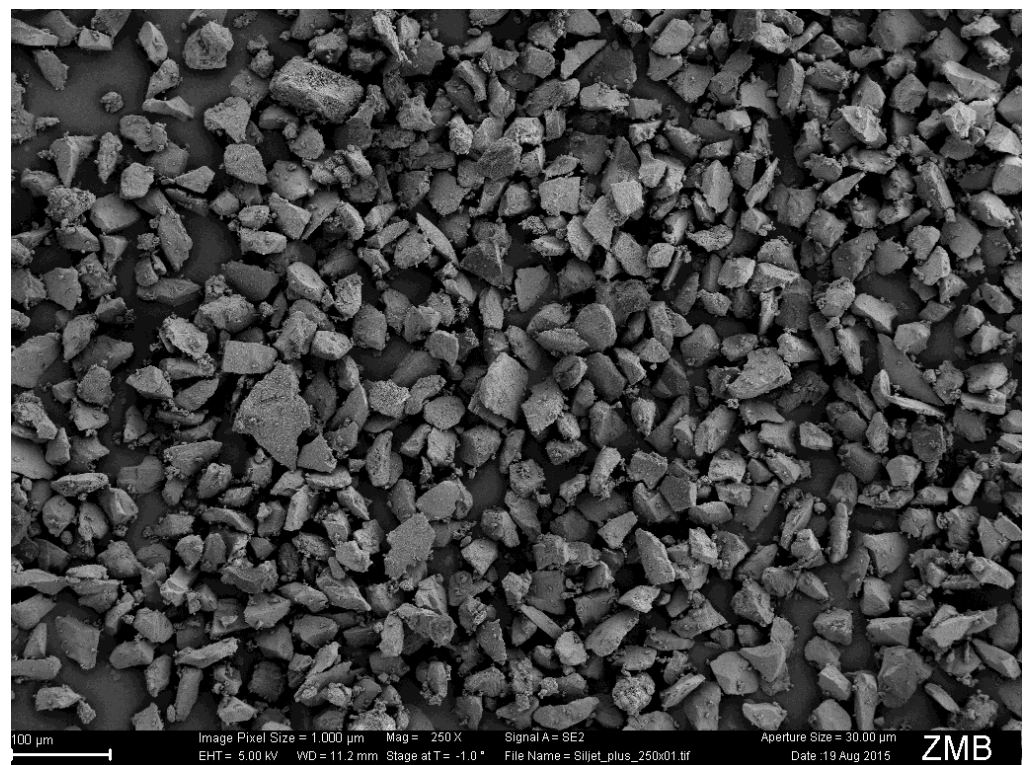

Figure 4c 